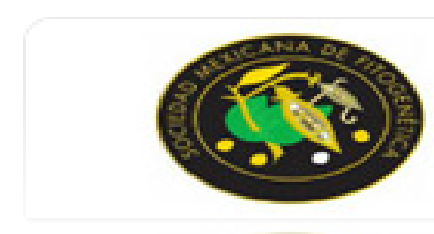

\section{Revista Fitotecnia Mexicana}

ISSN: 0187-7380

revfitotecniamex@gmail.com

Sociedad Mexicana de Fitogenética, A.C.

México

Moreno-Carrillo, Miguel A.; Hernández-Garay, Alfonso; Vaquera-Huerta, Humberto; Trejo-López, Carlos; Escalante-Estrada, José A.; Zaragoza-Ramírez, José L.; Joaquín-Torres, Bertín M. PRODUCTIVIDAD DE SIETE ASOCIACIONES Y DOS PRADERAS PURAS DE GRAMÍNEAS Y

LEGUMINOSAS EN CONDICIONES DE PASTOREO

Revista Fitotecnia Mexicana, vol. 38, núm. 1, 2015, pp. 101-108

Sociedad Mexicana de Fitogenética, A.C.

Chapingo, México

Disponible en: http://www.redalyc.org/articulo.oa?id=61035375013

Cómo citar el artículo

- Número completo

- Más información del artículo

- Página de la revista en redalyc.org

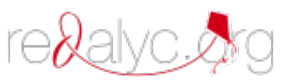

Sistema de Información Científica

Red de Revistas Científicas de América Latina, el Caribe, España y Portugal Proyecto académico sin fines de lucro, desarrollado bajo la iniciativa de acceso abierto 


\title{
PRODUCTIVIDAD DE SIETE ASOCIACIONES Y DOS PRADERAS PURAS DE GRAMÍNEAS Y LEGUMINOSAS EN CONDICIONES DE PASTOREO
}

\author{
FORAGE YIELD OF SEVEN ASSOCIATION AND TWO PURE SWARDS OF GRASSES \\ AND LEGUMES UNDER GRAZING CONDITIONS
}

\author{
Miguel A. Moreno-Carrilloํㅜ, Alfonso Hernández-Garay ${ }^{1 \star}$, Humberto Vaquera-Huerta ${ }^{1}$, Carlos Trejo-López ${ }^{1}$, \\ José A. Escalante-Estrada ${ }^{1}$, José L. Zaragoza-Ramírez ${ }^{2}$ y Bertín M. Joaquín-Torres ${ }^{3}$
}

\begin{abstract}
${ }^{1}$ Campus Montecillo, Colegio de Postgraduados. Km. 36.5 Carr. México-Texcoco. 56230, Montecillo, Texcoco, Edo. de México. ${ }^{2}$ Departamento de Zootecnia, Universidad Autónoma Chapingo. Km. 38.5 Carr. México-Texcoco. 56230, Chapingo,Texcoco, Edo. de México. ${ }^{3}$ Universidad del Papaloapan. Ferrocarril s/n. 68400, San Antonio, Loma Bonita, Oax.
\end{abstract}

${ }^{*}$ Autor para correspondencia (hernan@colpos.mx)

\section{RESUMEN}

Con el objetivo de encontrar la mejor asociación que maximice el rendimiento de materia seca del forraje, se estudiaron siete asociaciones de dos gramíneas (Dactylis glomerata L. y Lolium perenne L.) y una leguminosa (Trifolium repens $\mathrm{L}$.) en condiciones de pastoreo, en Texcoco, Estado de México. Se utilizaron siete asociaciones de trébol blanco (TB), ovillo $(\mathrm{O})$ y ballico perenne (BP) que fueron (en \%): 30:20:50 (TB:O:BP); 10:70:20 (TB:O:BP); 40:60 (TB:BP); 30:50:20 (TB:O:BP); 20:40:40 (TB:O:BP); 40:60 (TB:O) y 10:20:70 (TB:O:BP). Además se incluyeron dos praderas con gramíneas puras: 100 (BP); 100 $(O)$. Los nueve tratamientos se distribuyeron en un diseño de bloques completos al azar con cuatro repeticiones. Se evaluó el rendimiento de materia seca y la composición botánica. Las praderas fueron defoliadas por ovinos, cada cuatro semanas en primavera-verano y cada seis durante otoño-invierno. La asociación 10:20:70 (TB:O:BP) mostró el mayor rendimiento anual de materia seca, que superó en $66 \%$ a la asociación 10:70:20 (TB:O:BP) y a la pradera pura de ovillo $(P<0.05)$. Entre estaciones, el mayor rendimiento anual de materia seca se presentó en primavera-verano y el menor en otoño-invierno, con 69 y $31 \%$ del rendimiento anual. En promedio, ballico perenne, pasto ovillo y trébol blanco aportaron 47, 21 y $13 \%$ respectivamente, al rendimiento anual de forraje. Con base en el rendimiento anual de forraje y su distribución estacional, la mejor asociación fue 10:20:70 (TB:O:BP).

Palabras clave: Dactylis glomerata, Lolium perenne, Trifolium repens, composición botánica, rendimiento de forraje.

\section{SUMMARY}

To find the best association for maximizing dry matter yield, seven associations of two grasses (Dactylis glomerata $\mathrm{L}$. and Lolium perenne L.) and one legume (Trifolium repens $\mathbf{L}$.) were studied under grazing conditions at Texcoco, State of México. The associations of white clover (WC), orchard grass $(\mathrm{O})$, and perennial ryegrass (PR) were (in \%): 30:20:50 (WC:O:PR); 10:70:20 (WC:O:PR); 40:60 (WC:PR); 30:50:20 (WC:O:PR); 20:40:40 (WC:O:PR); 40:60 (WC:O); and 10:20:70 (WC:O:PR. Additionally, two pure swards with 100 (PR) and $100(\mathrm{O})$ were included. The nine treatments were distributed in a totally random block design with four replications. The evaluated variables were dry matter yield and botanical composition. Swards were defoliated by sheep every four weeks in the Spring-Summer, and every six weeks in the Fall-Winter. The 10:20:70 (WC:O:PR) association showed the highest annual dry matter yield, $66 \%$ more than the 10:70:20 (WC:O:PR) association and the pure orchard grass sward $(\mathrm{P}<0.05)$. Among seasons, the highest seasonal dry matter yield was recorded in the Spring-Summer, and the lowest in the Fall-Winter, with 69 and 31 $\%$ of the annual herbage yield. On average, perennial ryegrass, orchard grass, and white clover accounted for 47,21 , and $13 \%$ respectively, to total dry matter yield. Based on annual herbage yield and seasonal distribution, the best association was 10:20:70 (WC:O:PR).

Index words: Dactylis glomerata, Lolium perenne, Trifolium repens, botanical composition, herbage yield.

\section{INTRODUCCIÓN}

En México, más de $50 \%$ de la superficie nacional se dedica a la ganadería. El pastoreo se realiza en todos los estados del país y ocupa cerca de $62.5 \%$ de los 2, 000, 000 de $\mathrm{km}^{2}$ de tierra disponible (CONAGRO, 2006). Los forrajes constituyen una parte importante en la dieta de los rumiantes en las unidades ganaderas, y son la fuente más barata para la producción animal (Pérez et al., 2002). En la zona central del país existen sistemas de producción animal que tienen como componente importante el uso de praderas puras y asociadas. Según SIAP (2009), el rendimiento de pastos cultivados es de $19 \mathrm{t} \mathrm{MS} \mathrm{ha}^{-1}$.

El establecimiento de praderas puras o asociadas de mayor rendimiento y valor nutritivo de forraje permite disminuir los costos de producción (en comparación con el uso de dietas balanceadas) y asegurar una alta producción animal (Camacho y García, 2002). En estas explotaciones, el objetivo del manejo de praderas es mantener una alta y sostenida producción de forraje de buena calidad durante el año, la cual se puede lograr al evaluar el potencial de rebrote de las asociaciones de gramíneas y leguminosas presentes y su adaptación a las condiciones ambientales.

El clima tiene influencia directa en el crecimiento, desarrollo y rendimiento de las plantas. $\mathrm{Al}$ respecto, la tasa de 
crecimiento de cualquier especie forrajera es más sensible a la temperatura ambiental que la tasa de fotosíntesis y respiración, debido a que la temperatura interviene directamente en la aparición y expansión de la lámina foliar, aparición y muerte de tallos y estolones, así como en el crecimiento radical, por lo que las especies forrajeras logran la mayor producción de biomasa cuando se desarrollan en sus rangos óptimos de temperatura (Daly et al., 1996; McKenzie et al., 1999; Matthew et al., 2001; Lemaire, 2001).

El uso de leguminosas solas o asociadas con gramíneas permite mejorar el rendimiento, la distribución estacional y la calidad nutricional del forraje, que a su vez mejoran las ganancias de peso, producción de leche, y fertilidad del suelo por el aporte de nitrógeno atmosférico y una mejor intercepción de luz (Zaragoza et al., 2009). Al respecto, Sanderson et al. (2005) señalan que el rendimiento es menor en praderas puras o con dos especies que aquellas con más de tres. En la zona templada del país, trébol blanco (Trifolium repens L.), ballico perenne (Lolium perenne L.) y pasto ovillo (Dactylis glomerata L.), son especies que se emplean en condiciones de pastoreo debido a su lento establecimiento, buena cobertura del suelo, mejor crecimiento estacional y elevada producción de forraje (Castro et al., 2012).

El objetivo de este estudio fue determinar la mejor asociación de dos gramíneas y una leguminosa, sembradas en diferentes proporciones, en términos de rendimiento anual, distribución estacional, tasa de crecimiento, y composición botánica.

\section{MATERIALES Y MÉTODOS}

\section{Localización del área de estudio}

El estudio se realizó en una pradera asociada de trébol blanco variedad Ladino, pasto ovillo variedad Potomac y ballico perenne variedad Tetraploide Americano. Dicha pradera está ubicada en el Municipio de Texcoco, Estado de México. El suelo es franco arenoso, ligeramente alcalino (pH 7.8), con $2.4 \%$ de materia orgánica y se clasifica como Typic ustipsamments (Ortiz, 1997). El clima es templado subhúmedo con lluvias en verano, precipitación media anual de $645 \mathrm{~mm}$ y temperatura media anual de $15^{\circ} \mathrm{C}$; la menor temperatura promedio mensual es de $11.6^{\circ} \mathrm{C}$ y ocurre en enero, y la mayor en mayo con $18.4^{\circ} \mathrm{C}$ (García, 1988).

\section{Establecimiento de praderas}

La pradera se estableció en diciembre de 2009 y el experimento se llevó a cabo de marzo de 2010 a abril de 2011. Las gramíneas se sembraron en hileras separadas a $15 \mathrm{~cm}$; la leguminosa se sembró en forma perpendicular a las gramíneas, con una distancia entre hileras de $40 \mathrm{~cm}$. La densidad de siembra fue de $10 \mathrm{~kg} \mathrm{ha}^{-1}$ de pasto ovillo, $20 \mathrm{~kg} \mathrm{ha}^{-1} \mathrm{de}$ ballico perenne y $5 \mathrm{~kg} \mathrm{ha}^{-1}$ de trébol blanco.

Las asociaciones se distribuyeron en 36 parcelas experimentales de 7 × $8 \mathrm{~m}$, de acuerdo con un diseño de bloques al azar con cuatro repeticiones. Se bloqueó según la pendiente del terreno. Las praderas no se fertilizaron y en la época de estiaje se proporcionaron riegos a capacidad de campo cada dos semanas.

Antes de iniciar el experimento, en el mes de marzo de 2010, las praderas fueron defoliadas por ovinos, para uniformizarlas a una altura aproximada de $5 \mathrm{~cm}$ sobre el nivel del suelo. Posteriormente, la frecuencia de defoliación varió con la estación del año; cada cuatro semanas durante primavera-verano y cada seis en otoño-invierno, de acuerdo con las recomendaciones de Velasco et al. (2001; 2005), para pasto ovillo y ballico perenne en praderas puras. Para un mejor manejo de los ovinos, las praderas fueron delimitadas con cerco eléctrico.

\section{Variables medidas}

\section{Rendimiento de forraje}

En cada parcela se establecieron dos cuadros fijos de 0.25 $\mathrm{m}^{2}$, donde con tijeras se cortó el forraje presente antes del pastoreo a $5 \mathrm{~cm}$ de altura. Inmediatamente después, las praderas fueron defoliadas por ovinos a una altura promedio de $5 \mathrm{~cm}$. El material cosechado se lavó y secó en una estufa de aire forzado por 48 horas a $55^{\circ} \mathrm{C}$ y se pesó. La acumulación de forraje estacional y anual en cada asociación, se obtuvo al sumar el forraje cosechado en los cortes correspondientes a los meses de cada estación y en todos los meses del año, respectivamente.

\section{Composición botánica}

Para determinar la composición botánica, en el muestreo cercano a la mitad de cada estación, se tomó una submuestra de aproximadamente $20 \%$ la cual se separó por especie motivo de estudio (pasto ovillo, ballico perenne y trébol blanco), material muerto, otros pastos y maleza. Las especies se secaron en una estufa de aire forzado a $55^{\circ} \mathrm{C}$ durante $48 \mathrm{~h}$ y se pesaron.

\section{Análisis estadístico}

Para su análisis estadístico, los datos se agruparon de manera estacional y anual, conforme al diseño experimental ya indicado. Las medias de tratamientos se estimaron con el procedimiento LSMEANS y la comparación entre ellas por medio de la probabilidad de la diferencia (PDIF), con la prueba "t de Student" $(\mathrm{P} \leq 0.05)$, mediante 
el procedimiento estadístico PROC GLM del software SAS (SAS Institute, 2002).

\section{Datos climáticos}

Los promedios mensuales de temperatura a la intemperie (máxima, media y mínima) y la precipitación mensual durante el periodo de estudio, se obtuvieron de una estación meteorológica situada a $100 \mathrm{~m}$ del sitio experimental (Cuadro 1).

\section{RESULTADOS Y DISCUSIÓN}

\section{Rendimiento de forraje}

El mayor rendimiento anual (Cuadro 2) lo registró la asociación 10:20:70 de trébol blanco, pasto ovillo y ballico perenne (TB:O:BP) (12, $\left.611 \mathrm{~kg} \mathrm{MS} \mathrm{ha}^{-1}\right)$; en cambio, los menores rendimientos anuales correspondieron a la asociación 10:70:20 y a la pradera pura de ovillo, con 7612 y $7581 \mathrm{~kg}$ $\mathrm{MS} \mathrm{ha}^{-1}$, respectivamente. La asociación 10:20:70 (TB:O:BP) superó en $66 \%$ a la asociación 10:70:20 (TB:O:BP) y a la pradera pura de pasto ovillo. El rendimiento anual mostró el siguiente orden descendente: 10:20:70 (TB:O:BP) $>100$ (BP) > 20:40:40 (TB:O:BP) > 30:20:50 (TB:O:BP) > 40:60 $(\mathrm{TB}: \mathrm{BP})>40: 60(\mathrm{~TB}: \mathrm{O})>30: 50: 20(\mathrm{~TB}: \mathrm{O}: \mathrm{BP})>10: 70: 20$ $(\mathrm{TB}: \mathrm{O}: \mathrm{BP})>100(\mathrm{O})$ (Cuadro 2). En la asociación 10:20:70 (TB:O:BP), el aporte por especie fue: $70 \%$ de ballico perenne, $11 \%$ para trébol blanco y $8 \%$ del pasto ovillo; en la pradera pura el ballico perenne aportó $86 \%$.
En cuanto al rendimiento estacional (Cuadro 2), $69 \%$ del rendimiento anual de las nueve asociaciones se presentó en primavera y verano, y $31 \%$ en otoño e invierno; la estación de invierno tuvo el menor valor con $12 \%$ ( $<<0.01)$. En primavera el mayor rendimiento lo mostró la pradera pura de BP y la asociación 10:20:70 (TB:O:BP) $(\mathrm{P}<0.01)$ con 40 y $32 \%$ del rendimiento anual, estadísticamente diferentes a las asociaciones 10:70:20 (TB:O:BP), 30:50:20 (TB:O:BP), 40:60 (TB:O) y a la pradera pura de ovillo $(\mathrm{P}<0.01)$. Sin embargo, en verano, otoño e invierno no hubo diferencias estadísticas entre asociaciones.

Al evaluar praderas asociadas con más de dos especies, Sanderson et al. (2005) observaron que en la época húmeda no hubo diferencias en la producción de forraje entre asociaciones, con un promedio de $9800 \mathrm{~kg}^{\mathrm{de}} \mathrm{MS} \mathrm{ha}^{-1}$, pero durante la época de estiaje las praderas con menor número de especies asociadas (dos especies) produjeron menor cantidad de forraje que aquellas con más de 6 especies (4800 vs. $7600 \mathrm{~kg} \mathrm{MS} \mathrm{ha}^{-1}$ ). El estrés por sequía durante los meses de verano a menudo es la causa de que las gramíneas y leguminosas queden latentes (Hudson et al., 2010). Sin embargo, en este estudio todas las asociaciones, particularmente aquellas con más de $40 \%$ de pasto ballico perenne, registraron los mayores rendimientos durante primaveraverano, producto de las buenas condiciones de luz y temperatura (Cuadro 1) y a la falta de estrés hídrico por haberles suministrado riego.

La distribución promedio estacional del rendimiento en

Cuadro 1. Promedios mensuales de temperatura, precipitación y número de heladas registradas durante el periodo experimental. Estación meteorológica del Colegio de Posgraduados, Montecillo, Texcoco, Estado de México.

\begin{tabular}{|c|c|c|c|c|c|}
\hline \multirow{2}{*}{$\begin{array}{l}\text { Mes (marzo a diciembre de } \\
\text { 2010, enero a abril de 2011) }\end{array}$} & \multicolumn{3}{|c|}{ Temperatura $\left({ }^{\circ} \mathrm{C}\right)$} & \multirow{2}{*}{$\begin{array}{l}\text { Número de } \\
\text { heladas }\end{array}$} & \multirow{2}{*}{$\begin{array}{l}\text { Precipitación } \\
\quad(\mathrm{mm})\end{array}$} \\
\hline & Máxima & Mínima & Promedio & & \\
\hline Marzo & 32 & -3 & 15.3 & 4 & 15.2 \\
\hline Abril & 34 & 0 & 17.4 & 0 & 39.5 \\
\hline Mayo & 36 & -3 & 20 & 0 & 10.5 \\
\hline Junio & 36 & 4 & 20.7 & 0 & 49.2 \\
\hline Julio & 33.5 & 5.5 & 18.7 & 0 & 208.6 \\
\hline Agosto & 29 & 5 & 18 & 0 & 149.5 \\
\hline Septiembre & 29.5 & -4 & 17.3 & 2 & 44.3 \\
\hline Octubre & 30 & -4.5 & 13 & 14 & 0 \\
\hline Noviembre & 29.5 & -13 & 9.7 & 10 & 0 \\
\hline Diciembre & 23.4 & -6.4 & 8.5 & 2 & 0 \\
\hline Enero & 29 & -7.5 & 11.1 & 20 & 44 \\
\hline Febrero & 30 & -8 & 13.2 & 8 & 3 \\
\hline Marzo & 26.8 & 0.6 & 13.7 & 0 & 1.8 \\
\hline Abril & 36 & 2 & 19.6 & 0 & 12.2 \\
\hline
\end{tabular}


Cuadro 2. Rendimiento de forraje estacional y anual ( $\left.\mathrm{kg} \mathrm{MS} \mathrm{ha}^{-1}\right)$, de siete asociaciones y dos praderas puras de gramíneas y leguminosas.

\begin{tabular}{|c|c|c|c|c|c|c|c|c|c|c|c|c|}
\hline \multirow{3}{*}{$\begin{array}{l}\text { Estación } \\
\text { del año }\end{array}$} & \multicolumn{9}{|c|}{ Asociaciones } & \multirow{3}{*}{ EEM } & \multirow{3}{*}{ Sig } & \multirow{3}{*}{ Prom } \\
\hline & $30: 20: 50$ & $10: 70: 20$ & 100 & 100 & $40: 60$ & $30: 50: 20$ & $20: 40: 40$ & $40: 60$ & $10: 20: 70$ & & & \\
\hline & TB:O:BP & TB:O:BP & $\mathrm{BP}$ & $\mathrm{O}$ & TB:BP & TB:O:BP & TB:O:BP & TB:O & TB:O:BP & & & \\
\hline Primavera & $3120 \mathrm{ABab}$ & $2620 \mathrm{Ba}$ & $4329 \mathrm{Aa}$ & $2260 \mathrm{~B}$ & $3165 \mathrm{ABa}$ & $2528 \mathrm{Bab}$ & $3122 \mathrm{ABa}$ & $2367 \mathrm{Bab}$ & $4084 \mathrm{~A} \mathrm{a}$ & 183 & ** & $3066 \mathrm{a}$ \\
\hline Verano & $3756 \mathrm{a}$ & $2757 \mathrm{a}$ & $3867 \mathrm{ab}$ & 2523 & 3023 a & $3120 \mathrm{a}$ & $3430 \mathrm{a}$ & $3406 \mathrm{a}$ & $4312 \mathrm{a}$ & 141 & ns & $3355 \mathrm{a}$ \\
\hline Otoño & $1887 \mathrm{ab}$ & $1209 \mathrm{~b}$ & $1534 \mathrm{bc}$ & 1604 & $1984 \mathrm{ab}$ & $1440 \mathrm{ab}$ & $2051 \mathrm{ab}$ & $1759 \mathrm{~b}$ & $2767 \mathrm{ab}$ & 112 & ns & $1804 \mathrm{~b}$ \\
\hline Invierno & $1028 \mathrm{~b}$ & $1027 \mathrm{~b}$ & $1134 \mathrm{c}$ & 1194 & $1192 \mathrm{~b}$ & $926 \mathrm{~b}$ & $1201 \mathrm{~b}$ & $979 \mathrm{~b}$ & $1449 \mathrm{~b}$ & 40 & ns & $1126 \mathrm{c}$ \\
\hline Promedio & $2448 \mathrm{AB}$ & 1903 B & $2716 \mathrm{AB}$ & $1895 \mathrm{~B}$ & $2341 \mathrm{AB}$ & $2003 \mathrm{~B}$ & $2451 \mathrm{AB}$ & $2128 \mathrm{AB}$ & $3153 \mathrm{~A}$ & & $* *$ & \\
\hline EEM & 265 & 197 & 350 & 131 & 201 & 217 & 221 & 222 & 287 & & & 104 \\
\hline $\begin{array}{l}\text { Rendimiento } \\
\text { anual }\end{array}$ & $9790 \mathrm{AB}$ & $7612 \mathrm{~B}$ & $10,863 \mathrm{AB}$ & $7581 \mathrm{~B}$ & $9363 \mathrm{AB}$ & $8014 \mathrm{AB}$ & $9803 \mathrm{AB}$ & $8510 \mathrm{AB}$ & $12,611 \mathrm{~A}$ & & * & \\
\hline
\end{tabular}

Diferente literal mayúscula en la misma hilera, indica diferencias significativas entre asociaciones $(\mathrm{P}<0.01)$. Diferente literal minúscula en la misma columna, indica diferencias significativas entre estaciones del año para cada asociación $(\mathrm{P}<0.01)$. TB = trébol blanco, $\mathrm{O}=$ ovillo, $\mathrm{BP}=$ ballico perenne; $\mathrm{EEM}=$ error estándar de la media; $\mathrm{ns}=$ no significativo; $\mathrm{Sig}=$ significancia, ${ }^{*}=(\mathrm{P}<0.05),{ }^{* *}=(\mathrm{P}<0.01)$; Prom $=$ promedio.

las nueve asociaciones fue $33,36,19$ y $12 \%$ para primavera, verano, otoño e invierno $(\mathrm{P}<0.05)$, respectivamente; las mayores proporciones registradas en primavera y verano se atribuyen a que en estas dos estaciones se registraron las condiciones ambientales adecuadas, particularmente de temperatura, que permitieron a las tres especies manifestar su máximo potencial productivo (Cuadro 1). Al respecto, se ha señalado que al aumentar la temperatura aumenta la tasa de aparición y expansión foliar y aunada con un nivel adecuado de humedad, la pradera alcanza rápidamente su índice de área foliar óptimo en primavera y verano, como lo consignaron Velasco et al. (2001; 2005), para pasto ovillo y ballico perenne, y Clark et al. (1995) y Brock et al. (1989) para trébol blanco.

Al comparar la contribución de cada especie al rendimiento anual (Cuadro 3), en todas las asociaciones el ballico perenne aportó más de $50 \%$; destaca que la época de primavera-verano concentró $80 \%$ del rendimiento anual, mientras que en invierno la aportación fue de apenas $6 \%$. La pradera pura de BP con $9286 \mathrm{~kg} \mathrm{MS} \mathrm{ha}^{-1}$ presentó el mayor rendimiento anual al superar en 172 y $193 \%$ a las asociaciones con $20 \%$ de esta especie.

Al respecto, Matthew et al. (2001) y Lemaire (2001) señalan que el rendimiento de las plantas forrajeras está sujeto a un patrón de crecimiento influenciado por la temperatura, humedad y nutrientes, que determinan la cantidad de biomasa por ciclo de producción, y el rendimiento estacional y anual. En este estudio, tales condiciones se presentaron durante primavera-verano. Por su parte, Pérez et al. (2002) señalan que para maximizar la acumulación de forraje en el tiempo, la energía solar debe ser interceptada por las hojas fotosintéticamente activas, a través de periodos de crecimiento activo.
Por ello, entre mayor sea el índice de área foliar de una pradera, mayor será la proporción de radiación incidente interceptada por la masa foliar y, durante el rebrote la tasa de crecimiento del forraje aumenta hasta que $95 \%$ de la luz incidente es interceptada. Tales condiciones se registraron en primavera y verano. Otro factor que permitió a que el ballico perenne fuese la especie predominante es que tanto el pasto ovillo como el trébol blanco son de establecimiento lento, además de que el pasto ovillo es de porte menos erecto que el pasto ballico perenne (Brock et al., 1989; Durand et al., 1999).

El pasto ovillo registró menor rendimiento que el ballico solo y que las asociaciones, y el ovillo puro tuvo un rendimiento anual de $7581 \mathrm{~kg} \mathrm{MS} \mathrm{ha}^{-1}$ (Cuadro 3). En promedio de las asociaciones, el mayor rendimiento fue durante verano con $46 \%$ del anual $(\mathrm{P}<0.01)$. La pradera pura de ovillo y la asociación 10:20:70 (TB:O:BP) con 4553 y $961 \mathrm{~kg} \mathrm{MS} \mathrm{ha}^{-1}$ fueron las que presentaron el mayor y menor rendimiento de pasto ovillo, respectivamente $(\mathrm{P}<0.01)$.

La escasa contribución del ovillo al rendimiento anual pudo deberse a que cuando esta especie es sometida a una severa defoliación, el número de hojas remanentes es mínimo por lo que su rebrote es lento. De acuerdo con Turner et al. (2007), para asegurar un crecimiento vigoroso del pasto ovillo se recomienda dejar cuatro hojas remanentes por tallo y un intervalo de defoliación adecuado para maximizar el rebrote y la persistencia.

Zaragoza et al. (2009) evaluaron el efecto de la frecuencia de pastoreo (28 y 35 d durante primavera, verano y otoño; y en invierno de 35 a $42 \mathrm{~d}$ ), con tres alturas de forraje residual, pastoreo severo (3 a $6 \mathrm{~cm})$, intermedio $(7 \mathrm{a} 10 \mathrm{~cm})$ y ligero (11 a $14 \mathrm{~cm}$ ) en una asociación de alfalfa-ovillo. Estos 


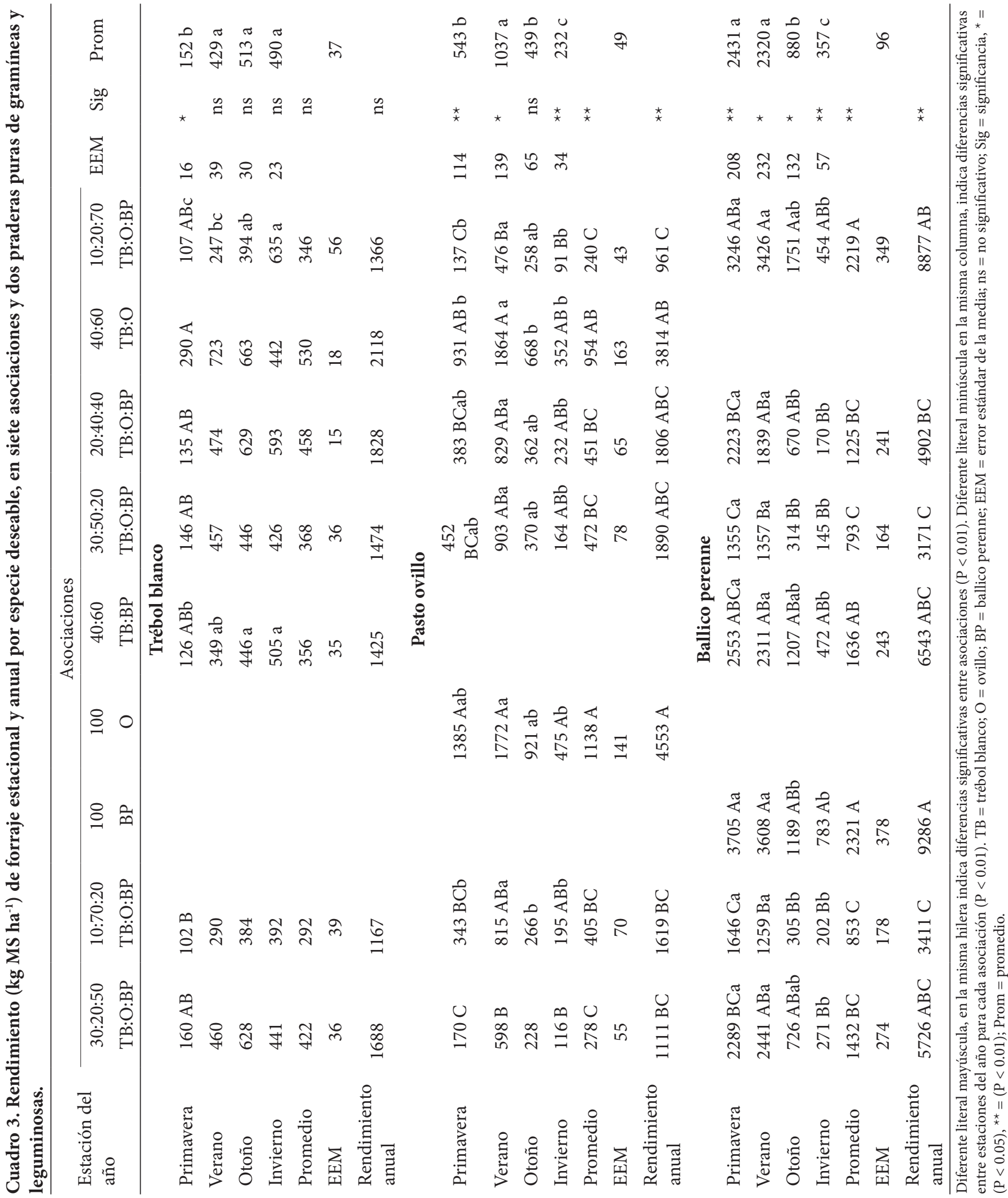


autores encontraron que los pastoreos severos favorecen el rendimiento de alfalfa (Medicago sativa $\mathrm{L}$.) y reducen el de pasto ovillo, así como la presencia de malezas; en contraste, las defoliaciones ligeras promueve el mayor rendimiento del pasto ovillo y mayor acumulación de material muerto; además reportaron que el pastoreo ligero y poco frecuente incrementa la altura de la pradera, peso, densidad de tallos y área foliar del pasto ovillo, mientras que los pastoreos severos y frecuentes favorecen a la alfalfa.

En la mayoría de las asociaciones estudiadas el trébol blanco fue la especie que presentó la menor aportación al rendimiento anual de forraje, con un promedio por asociación de $1581 \mathrm{~kg} \mathrm{MS} \mathrm{ha-1}$, sin diferencias estadísticas entre asociaciones en el verano, otoño e invierno $(\mathrm{P}>0.05)$. Al comparar el rendimiento de esta especie en cada asociación entre estaciones, se detectó un incremento progresivo conforme transcurrieron las estaciones del año de primavera a invierno, significativo en las asociaciones 40:60 (TB:BP) y 10:20:70 (TB:O:BP) $(\mathrm{P}<0.05)$. En contraste con lo observado en las gramíneas, el trébol blanco registró la menor aportación al rendimiento anual durante primavera-verano, con $37 \%$ del total.

La escasa aportación del trébol blanco en comparación con las gramíneas, se pueden deber a que los datos del presente estudio corresponden al primer año de la pradera. Su lento establecimiento y las condiciones ambientales predominantes durante la siembra, principalmente la temperaturas bajas, no estimularon el crecimiento del trébol en las praderas asociadas, según reportaron Brock et al. (1989).

Los resultados obtenidos en el presente estudio no concuerdan con lo señalado por Castro et al. (2012) quienes al evaluar cinco asociaciones de ballico perenne, pasto ovillo y trébol blanco en el Valle de México, observaron el mayor rendimiento de forraje $\left(17,270 \mathrm{~kg} \mathrm{MS} \mathrm{ha}^{-1}\right)$ en una pradera con proporciones 40:20:40 (TB:O:BP) de cada especie, donde la aportación al rendimiento anual de trébol blanco fue de 10,000 kg MS ha-1. Tampoco concuerdan con lo reportado por Brock et al. (1989), los que indicaron que la mayor producción del trébol blanco con respecto a las gramíneas, fue durante primavera-verano, y se debió a que las condiciones ambientales, principalmente la temperatura óptima, estimulan el crecimiento del trébol en praderas puras y asociadas. En nuestro estudio las bajas temperaturas que prevalecieron al momento de sembrarlo (Cuadro 1), pudieron haber afectado más al trébol blanco, en especial por ser una especie de lento establecimiento.

La intensidad de pastoreo (a $5 \mathrm{~cm}$ de altura) pudo ser el factor que permitió al trébol blanco que fuera la especie dominante durante el periodo de otoño-invierno en la mayoría de las siete asociaciones, ya que por tener un hábito de crecimiento estolonífero es menos susceptible a perder meristemos de crecimiento que las especies erectas como ballico perenne y ovillo, como lo reportó Hodgson (1990). Adicionalmente, se considera que el arreglo horizontal de sus hojas permitió a las plantas de trébol blanco restablecer su área foliar y utilizar la luz solar más rápido durante el invierno, que el ballico perenne y el pasto ovillo (Brock et al., 1989).

La mayor aportación del ballico perenne al rendimiento anual, particularmente en primavera y verano, se debió a que la temperatura ambiental osciló alrededor de las temperaturas óptimas $\left(18.7^{\circ} \mathrm{C}\right.$; Cuadro 1) para el crecimiento de esta especie (Black et al., 2006). Las primeras etapas de desarrollo son clave para el establecimiento exitoso de los pastos en praderas, ya que la germinación y emergencia son importantes en la capacidad competitiva porque determinan en gran medida el potencial productivo (Durán et al., 2011).

En invierno se registró el menor rendimiento de las dos gramíneas evaluadas, debido a las bajas temperaturas que llegaron a valores bajo cero; de las cuatro estaciones, las temperaturas mínimas más bajas se presentaron en otoño e invierno (Cuadro 1). Al respecto, las bajas temperaturas, aún en periodos cortos, disminuyen el crecimiento de los pastos por lo que se pueden presentar tasas de acumulación de forraje muy bajas (Hudson et al., 2010).

\section{Composición botánica}

En general, el ballico perenne contribuyó al rendimiento total de forraje con $47 \%$, el pasto ovillo con $21 \%$ y el trébol blanco con $13 \%$, mientras que el $19 \%$ restante lo integró el material muerto, otros pastos y malezas (Figura 1). La contribución de cada especie al rendimiento en las praderas puras fue de 86 y $60 \%$ para el ballico perenne y pasto ovillo, mientras que en asociaciones su aportación fue muy variable. El ballico perenne fue la especie que más aportó, con porcentajes que variaron de 70 a $40 \%$ en las asociaciones 40:60 (TB:BP) y 30:50:20 (TB:O:BP), respectivamente (Figura 1), con una diferencia de $3372 \mathrm{~kg} \mathrm{MS} \mathrm{ha}^{-1}$ (Cuadro 4). El trébol blanco en las asociaciones 40:60 (TB:O) y 10:20:70 (TB:O:BP) contribuyó con 25 y $11 \%$, respectivamente (752 $\mathrm{kg} \mathrm{MS} \mathrm{ha}^{-1}$, de diferencia). El pasto ovillo registró una diferencia de $2853 \mathrm{~kg} \mathrm{MS} \mathrm{ha}^{-1}$ entre las asociaciones 40:60 (TB:O) y 10:20:70 (TB:O:BP).

En la asociación 10:20:70 (TB:O:BP), el ballico perenne aportó $70 \%$, seguido del trébol blanco con $11 \%$, y el ovillo con $8 \%$. En las asociaciones 40:60 de trébol blanco con pasto ovillo (TB:O) y ballico perenne (TB:BP), las aportaciones de material muerto, maleza y otros pastos fueron de 30 y $15 \%$, respectivamente. En las asociaciones de las tres 


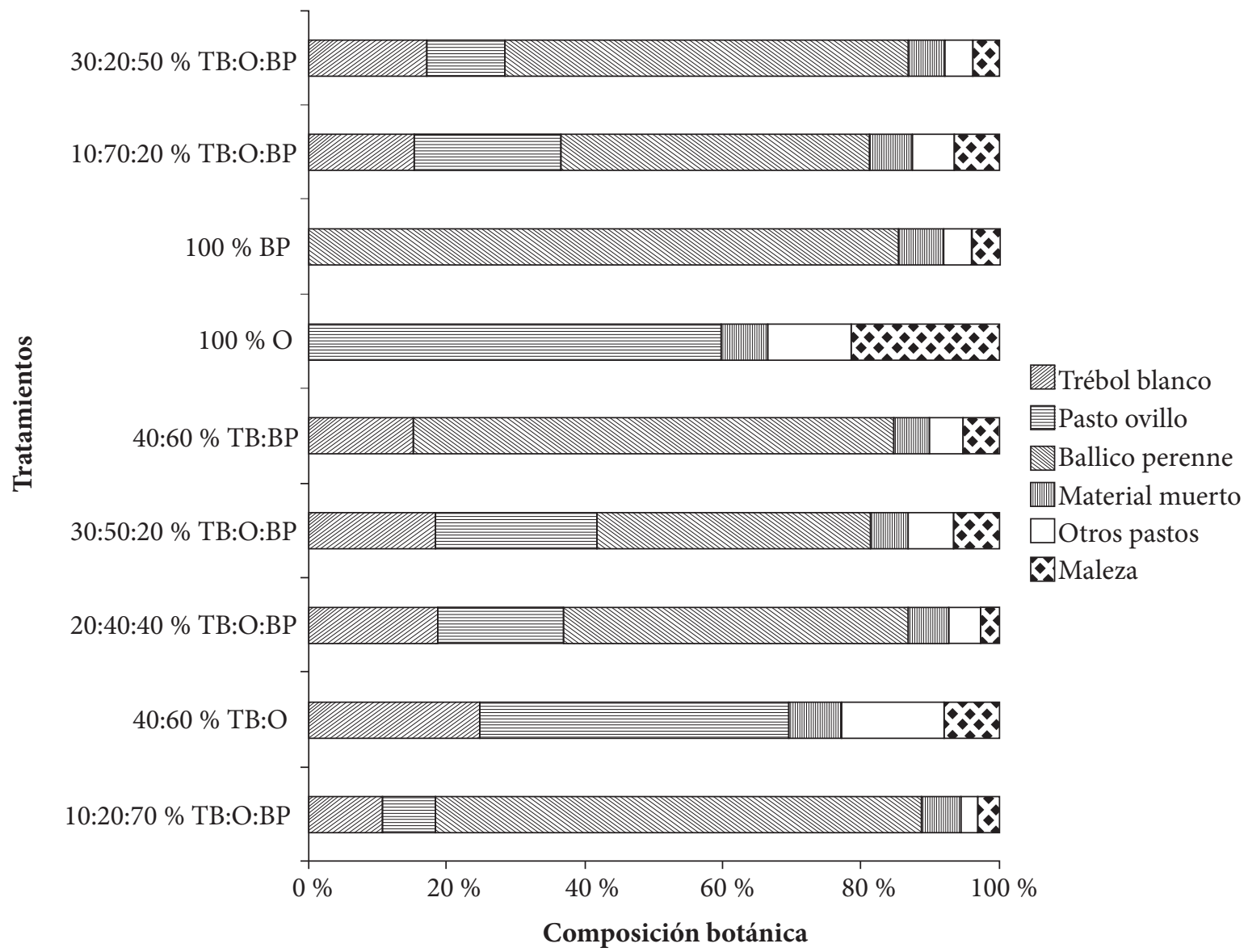

Figura 1. Porcentajes promedios de las especies deseables que integran las siete asociaciones y dos praderas puras de gramíneas y leguminosas.

especies con mayores cantidades de pasto ovillo, 10:70:20 y 30:50:20 (TB:O:BP), la proporción de material muerto, maleza y otros pastos fue mayor de $15 \%$. En contraste, cuando hubo la mayor cantidad de ballico perenne en la asociación, 30:20:50 y 10:20:70 (TB:O:BP), el porcentaje de esos componentes fue menor a $15 \%$. Las praderas puras de ovillo y ballico perenne registraron 40 y $15 \%$ de componentes no deseables, respectivamente (Figura 1).

Estos resultados no concuerdan con los registrados por Castro et al. (2012) en cinco asociaciones de gramíneas y leguminosas en el Valle de México, pues ellos observaron que el trébol blanco contribuyó con $50 \%$ al rendimiento total de forraje, el ballico perenne con $28 \%$ y el pasto ovillo con $12 \%$; el restante $10 \%$ estuvo integrado por material muerto, otros pastos y maleza. La falta de concordancia pudo deberse a que Castro et al. (2012) evaluaron praderas con 2 años de establecidas, mientras que las praderas de nuestro estudio tenían menos de un año cuando se inició la evaluación.

La intensidad de pastoreo usado en esta investigación tal vez redujo la productividad del pasto ovillo y por ello se au- mentó la invasión de malezas, como reportaron Coleman y Sollenberger (2007), también se elevó la proporción de otros pastos, como lo muestran la pradera pura y las asociadas con mayor proporción de ovillo (Figuras 1).

\section{CONCLUSIONES}

Con base en el rendimiento anual de forraje, la mejor asociación fue 10:20:70 (TB:O:BP). Durante primavera-verano la pradera pura de ballico perenne y la asociación 10:20:70 (TB:O:BP) registraron el mayor rendimiento de forraje. El ballico perenne fue la especie dominante y contribuyó con $47 \%$ al rendimiento anual, seguido del pasto ovillo con 21 $\%$ y, en menor proporción el trébol blanco con $13 \%$.

\section{AGRADECIMIENTOS}

Al Consejo Nacional de Ciencia y Tecnología por el apoyo brindado para la realización de los estudios de doctorado de Miguel Ángel Moreno Carrillo, y a la Línea 11 Sistemas de Producción Agrícola, Pecuaria, Forestal y Acuícola por el apoyo brindado para la realización de esta investigación. 


\section{BIBLIOGRAFÍA}

Black A. D., D. J. Moot and R. J. Lucas (2006) Development and growth characteristics of Caucasian and white clover seedlings, compared with perennial ryegrass. Grass Forage Science 61:442-453.

Brock J. L., J. R. Caradus and M. J. M Hay (1989) Fifty years of white clover research in New Zealand. Proceedings of the New Zealand Grassland Association50:25-39.

Camacho G. J. L. y J. G. García M. (2002) Producción y calidad del forraje de cuatro variedades de alfalfa asociadas con trébol blanco, ballico perenne, festuca alta y pasto ovillo. Veterinaria México 34:149-177.

Castro R. R., A Hernández G., J. Pérez P., J. Hernández G., A. R. Quero C., J. F. Enríquez Q. y P. A. Martínez H. (2012) Comportamiento productivo de cinco asociaciones gramíneas-leguminosas bajo condiciones de pastoreo. Revista Fitotecnia Mexicana 35:87-95.

Clark H., P. C. D. Newton, C. C. Bell and E. M. Glasgow (1995) The influence of elevated $\mathrm{CO}_{2}$ and simulated seasonal changes in temperature on tissue turnover in pasture turfs dominated by ryegrass (Lolium perenne L.) y white clover (Trifolium repens L.). Journal of Applied Ecology 32:128-136.

Coleman S. W. and L. E. Sollenberger (2007) Plant-herbivore interactions. In: R. F. Barnes et al. (ed.) Forages: The Science of Grassland Agriculture, Vol. 2. 6th ed. Blackwell Publ., Ames, IA. pp:123-136.

Daly M. J., R. M. Hunter, G. N. Green and L. Hunt (1996) A comparison of multi-species pasture with ryegrass-white clover pastures under dry land conditions. Proceedings of the New Zealand Grassland Association 58:53-58.

Durand J. L., R. Schäufele and F. Gastal (1999) Grass leaf elongation rate as a function of developmental stage and temperature: Morphological analysis and modeling. Annals of Botany 83:577-588.

Durán P. N., J. A. Ruiz C., D. R. González E., G. Núñez H., F. J. Padilla R. y S. H. Contreras R. (2011) Temperaturas cardinales de desarrollo en la etapa siembra-emergencia de 11 pastos forrajeros. Revista Mexicana de Ciencias Pecuarias 2:347-357.

García E. (1988) Modificaciones al sistema de clasificación climática de Köppen. $4^{\text {a }}$ ed. Universidad Nacional Autónoma de México. México, D. F. 217 p.

Hodgson J. (1990) Grazing Management: Science into Practice. Longman Scientific \& Technical. Harlow, England. 204 p.

Hudson D. J., R. H. Leep, T. S. Dietz and A. Kravchenko (2010) Integrated warm and cool season grass and legume pastures: I. Seasonal forage dynamics. Agronomy Journal 102:303-309.

Lemaire G. (2001) Ecophysiology of grasslands Aspects of forage plant populations in grazed swards. In: Proc. XIX International Grassland Congress. Brazilian Society of Animal Husbandry, Sociedad Brasileira de Zootecnia (eds.). São Pedro, São Paulo. Brasil. pp:29-37.

Matthew C. G., E. N. Val Loo, E. R. Tom, L. A. Dawson and D. A. Care (2001) Understanding shoot and root development. In: Proc. XIX International Grassland Congress. Brazilian Society of Animal Husbandry, Sociedad Brasileira de Zootecnia (eds.). São Pedro, São Paulo, Brasil. pp:19-27.

McKenzie B. A., P. D. Kemp, D. J. Moot, C. Matthew and R. J. Lucas (1999) Environmental effects on plant growth and development. In: New Zealand Pasture and Crop Science. J. White and J. Hodgson (eds.). Auckland, N. Z. Oxford University. pp:29-44.

Ortiz S. C. (1997) Colección de monolitos. Montecillo, Texcoco, Edo. México. México: Depto. Génesis de Suelos. Edafología, IRENAT. Colegio de Postgraduados. $17 \mathrm{p}$.

Pérez B. M. T., A. Hernández G., J. Pérez P., J. G Herrera H., R. Bárcena G. (2002) Respuesta productiva y dinámica de rebrote del ballico perenne a diferentes alturas de corte. Técnica Pecuaria en México 40:251-263.

SIAP, Servicio de Información y Estadística Agroalimentaria y Pesquera (2009) Producción Agrícola en México. Centro de Estadística Agropecuaria. http://www.siap.gob.mx/. (Enero 2012).

Sanderson M. A., K. J. Soder, L. D. Muller, K. D. Klement, R. H. Skinner and S. C. Goslee (2005) Forage mixture productivity and botanical composition in pastures grazed by dairy cattle. Agronomy Journal 97:1465-1471.

SAS Institute (2002) SAS User's Guide: Statistics (version 9.0 ed.). Cary NC, USA: SAS Inst. Inc.

Turner L. R., D. J. Donaghy, P. A. Lane and R. P. Rawnsley (2007) Distribution of water-soluble carbohydrate reserves in the stubble of prairie grass and orchard grass plants. Agronomy Journal 99:591-594.

Velasco Z. M. E., A. Hernández G., V. A. González H., J. Pérez P., H. Vaquera H., A. Galvis S. (2001) Curva de crecimiento y acumulación estacional del pasto ovillo (Dactylis glomerata L.) Técnica Pecuaria en México 39:1-14.

Velasco Z. M. E., A. Hernández G. and V. A. González H. (2005) Rendimiento y valor nutritivo de ballico perenne (Lolium perenne L.) en respuesta a la frecuencia de corte. Técnica Pecuaria en México 43:274-258.

Zaragoza E. J., A. Hernández G., J. Pérez P., J. G. Herrera H., F. Osnaya G., P. A. Martínez H., S. González M. and A. R. Quero C. (2009) Análisis de crecimiento estacional de una pradera asociada alfalfa-pasto ovillo. Técnica Pecuaria en México 47:173188. 